\title{
Implementación de sistemas de simulación para el entrenamiento como eje fundamental en la educación militar ${ }^{1}$
}

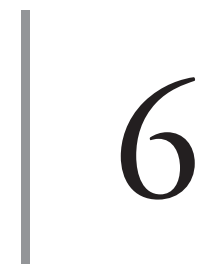

https://doi.org/10.21830/9789585380202.06

\author{
Cristhian Camilo Garcia Rodríguez \\ Daniel Felipe Molina Martínez ${ }^{3}$ \\ Andrés Alberto Ramirez Duque ${ }^{4}$ \\ Escuela Militar de Cadetes "General José María Córdova” \\ Daniel Alfonso Botero Rosas 5 \\ Universidad de La Sabana
}

\begin{abstract}
Resumen. El desarrollo de sistemas de entrenamiento utilizando tecnologías de simulación es un tema de interés en el Ejército Nacional de Colombia. Estos desarrollos se han orientado en las escuelas de formación a fortalecer los métodos de enseñanza utilizados en el entrenamiento militar, lo cual le permite al instructor evaluar y retroalimentar de una manera más didáctica y eficiente a los alumnos, así como reducir los costos asociados al entrenamiento convencional. En este capítulo se presentan los avances en el desarrollo de sistemas de entrenamiento en procedimientos tácticos en un ambiente de operaciones simulado realizados en la Escuela Militar de Cadetes "General José María
\end{abstract}

1 Este capítulo forma parte de los resultados de investigación del Grupo de Investigación en Ingeniería y Simulación (GINSI), categorizado en $\mathrm{C}$ por Minciencias y con código de registro COL0171262, del Centro de Investigación y Desarrollo en Simulación (CIDsi) de la Escuela Militar de Cadetes "General José María Córdova”, Bogotá, D. C., Colombia. Los puntos de vista que se presentan en este capítulo pertenecen a los autores y no reflejan necesariamente los de las instituciones participantes.

2 Ingeniero mecatrónico de la Universidad San Buenaventura. Estudiante de la Maestría en Comercio Internacional de la Universidad Sergio Arboleda. OrCID: https://orcid.org/0000-0001-7351-8132 - Contacto: cristhian.garcia@esmic.edu.co

3 Magíster y especialista en Gestión y Gerencia de Proyectos de la Universidad Ean. Ingeniero mecatrónico de la Universidad Agraria. ORCID: https://orcid.org/0000-0002-7926-1822 - Contacto: daniel. molinam@esmic.edu.co

4 Doctor en Ingeniería Eléctrica y magíster en Automatización Industrial de la Universidad Federal do Espirito Santo, Brasil. Ingeniero mecatrónico de la Universidad Nacional de Colombia. Orcid: https://orcid.org/0000-0002-8419-9285 - Contacto: andres.ramirezdu@esmic.edu.co_

5 Doctor y magíster en Ingeniería Biomédica de la Universidad Federal de Río de Janeiro. Médico de la Escuela Colombiana de Medicina. Orcid: https://orcid.org/0000-0002-2590-0756 - Contacto: daniel.botero@unisabana.edu.co 
Córdova" (ESMIC). Estos sistemas están orientados al desarrollo de las habilidades de precisión de tiro y liderazgo y toma de decisiones, necesarias en la formación del cadete para el adecuado desarrollo de las actividades misionales que se le asignen en el área de operaciones.

Palabras clave: educación militar; Ejército Nacional de Colombia; interfaz gráfica; tecnología; sistemas y niveles de enseñanza; simulaciones; software.

\section{Introducción}

Los nuevos retos de las escuelas de formación del Ejército de Colombia (Ciro \& Correa, 2014) requieren que el entrenamiento y reentrenamiento se realice de forma efectiva y eficiente. Esto se relaciona con el aumento de los ejercicios prácticos, la disminución de costos para el entrenamiento, el aumento del control del desempeño del estudiante, la disminución de los riesgos de accidentalidad y la integración de tecnologías que eviten el desplazamiento de personal hacia áreas de instrucción en el terreno. Estas necesidades se encuentran enmarcadas en los requerimientos de mando tipo misión para generar entrenamiento en operaciones en ambiente operacionales (OE, por su sigla en inglés) de fuerza, la cual está alineada con el Plan Estratégico para el Sector Defensa 2018-2022, orientado a continuar con el proceso de transformación, modernización y fortalecimiento institucional del sector defensa y seguridad de manera sostenible (Ejército Nacional de Colombia, 2013, 2015).

El presente trabajo se enfoca en identificar las falencias en los procesos de aprendizaje y en las prácticas de las anteriores habilidades mencionadas, la necesidad de implementar medios tecnológicos de simulación que puedan contribuir al entrenamiento y presentar los sistemas tecnológicos que se han desarrollado en la Escuela Militar de Cadetes "General José María Córdova" (ESMIC) (Roselli, 2011) para así finalmente, mediante pruebas de funcionalidad, analizar su aceptación por parte del usuario final.

\section{Marco teórico}

Atendiendo esta necesidad de las escuelas de formación del Ejército Nacional de Colombia se han desarrollado diferentes iniciativas mediante la implementación de proyectos de investigación que hacen uso de tecnologías de 
simulación (Margalef \& Arenas, 2006). Entre estas se destacan: la Escuela de Caballería (ESCAB) con la Iniciativa VESTA, orientada al manejo de torretas de vehículos de combate (Brash 3D, 2019); la Escuela de Artillería con el simulador de tiro parabólico, y los proyectos de la Escuela de Armas Combinadas con los juegos de guerra de los Cursos Comando e Intermedio. Por su parte, en la ESMIC se ha venido desarrollando la iniciativa AsIMOv, cuyo propósito es impulsar la investigación científica y el desarrollo tecnológico al servicio de la Fuerza (Giroux, 1997), de tal manera que se aporte al entrenamiento militar integral mediante el desarrollo de herramientas tecnológicas y sistemas de simulación. Las propuestas de investigación de esta iniciativa se centran en dos habilidades fundamentales que deben ser desarrolladas por los cadetes en formación: precisión en tiro, liderazgo y toma de decisiones.

\section{Métodos}

Para el presente desarrollo se establecieron tres actividades principales: recolección y análisis de información (Slavin, 2002), diseño de sistema de simulación e interoperabilidad. Estas se utilizaron tanto para el entrenamiento orientado a fortalecer y evaluar la precisión de tiro, como el liderazgo y la toma de decisiones (figura 1), presentando en cada uno los resultados obtenidos.

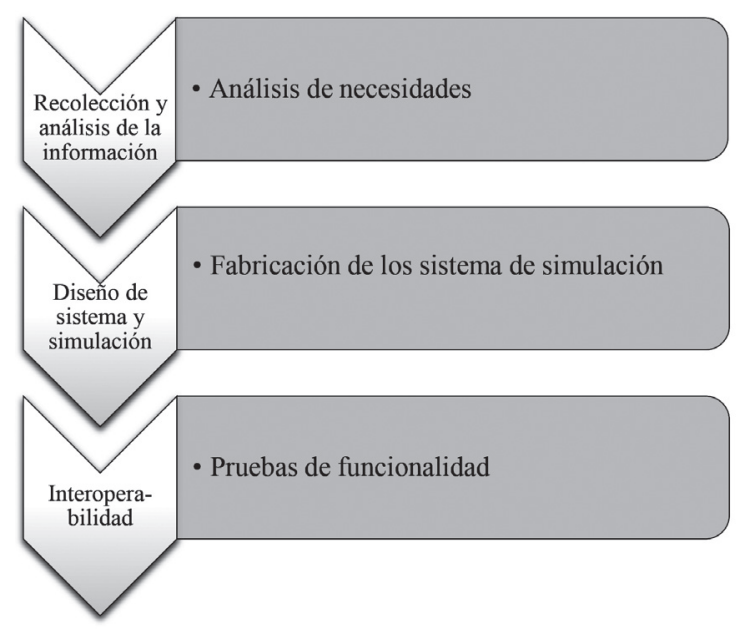

Figura 1. Metodología del desarrollo de la investigación.

Fuente: Elaboración propia. 


\section{Recolección y análisis de información}

El objetivo es identificar las necesidades de entrenamiento y reentrenamiento del personal que integra la Fuerza. Como bien se conoce, para mantener las capacidades y desarrollar nuevas habilidades en las operaciones militares es necesario emplear nuevas tecnologías que aporten al desarrollo profesional de oficiales, suboficiales y soldados profesionales, por lo tanto se realizó una búsqueda de la tecnología actual que se emplea en el entrenamiento colombiano para analizar su eficiencia, resultados y unidades que ponen en práctica dichos ejercicios. Con esta información se busca dar respuesta a las principales técnicas de entrenamiento que deben ser optimizadas, lo cual demuestra que los desarrollos de investigación de las escuelas de formación son una solución para estudiar y validar tecnología militar que aporta al entrenamiento (Ejército Nacional de Colombia, 2009).

En la recolección y análisis de la información se enfocó el área de estudio en las escuelas de formación, ya que todo el personal militar, como parte de su profesionalización, debe realizar cursos que garantizan el mejoramiento constante de sus habilidades. Con este propósito se desarrollan sistemas de simulación que permitan a los estudiantes entrenar en entornos similares a los que encuentran en el área de operaciones.

\section{Diseño de sistema de simulación}

Para el desarrollo del sistema y evaluación de tiro se parte de los principios de funcionamiento de los simuladores de tiro que actualmente se encuentran implementados en la EsMic, los cuales consisten en un sistema inalámbrico que funciona a través de un sensor magnético que se activa cuando se acciona el gatillo, de manera que simula el disparo de un fusil galil $5.56 \mathrm{~mm}$. El dispositivo láser instalado en el arma se activa e impacta el objetivo, que es una pantalla de cristal líquido que permite evaluar la precisión del disparo de los estudiantes. En la investigación, además, se tienen en cuenta las principales falencias de estos sistemas y las necesidades identificadas en la fase de recolección y análisis de la información.

Por otra parte, para el desarrollo del sistema de evaluación de liderazgo y toma de decisiones se parte de la caracterización de la respuesta fisiológica 
en ambientes de realidad virtual y la propuesta inicial de una herramienta de apoyo a la toma de decisiones, la cual se complementa con el sistema de entrenamiento militar en entornos virtuales implementado en la ESMIC, y para ello se propone el uso de una interfaz cerebro-computadora (BCI, por su sigla en inglés $)^{6}$ (Elbit Systems, 2020). El método más ampliamente adoptado es la implementación de un sistema BCI no invasivo a través del espectro de aplicación de la electroencefalografía $(\mathrm{EEG})^{7}$ (Kenneth \& Julie, 2017). En la EEG basada en BCI, la señal es recogida por una matriz de electrodos colocado en el cuero cabelludo (Bera, 2015).

\section{Interoperabilidad}

Se establecen protocolos de funcionalidad para los dos sistemas desarrollados: para el sistema de evaluación de precisión de tiro se realizaron ejercicios de tiro en una práctica, con un tiempo estimado de ocho horas al día. El paso a paso de las actividades realizadas se destaca a continuación:

1. Armonización: mediante la longitud de onda del láser y tomando un punto fijo en la mira, se ajusta el alcance del láser tanto en eje $\mathrm{X}$ y eje $\mathrm{Y}$ mediante rotaciones a los tornillos auxiliares.

2. Cargar y reconocer cada polígono: con el usuario de activación, determinar el reconocimiento $\mathrm{RF}^{8}$ de los dispositivos.

3. Realizar los tres tiros de prueba que proporciona el programa de disparo polígono láser portátil que ayudan a corroborar la armonización.

4. Realizar el ejercicio de disparo en el polígono.

5. Mostrar los resultados de la prueba y la estadística que proporciona el sistema para que el usuario tenga una realimentación cuantitativa y determinar así la aceptación por parte del usuario.

6 BcI: Brain Computer Interfaces ('interfaz cerebro computadora'), tecnología para la adquisición de ondas cerebrales, que posteriormente son analizadas a través de un software.

7 EEG: electroencefalografía, registro de la actividad bioeléctrica del cerebro.

8 RF: Radiofrecuencia. 
Asimismo, para el sistema de evaluación de liderazgo y toma de decisiones se propuso el siguiente protocolo:

1. Se explica al sujeto de investigación en forma clara el proyecto de investigación, así como el procedimiento que se va a realizar. Se obtiene la respectiva firma del consentimiento informado.

2. Se recolecta la actividad eléctrica cerebral del sujeto de investigación. Las señales de electroencefalografía (EEG) serán adquiridas mediante el casco para recolección de señales, el cual se conecta con el dispositivo 16-Channel Biosensing Board - Cyton-DAISY.

3. Es necesario identificar la señal relacionada con la variabilidad de la frecuencia cardiaca del individuo, por lo cual se utiliza el sensor Polar V800.

4. Se realiza la configuración del sistema de simulación de realidad virtual con el cual cuenta la Esmic.

5. El sujeto de investigación experimentará un ambiente de realidad virtual con fines introductorios al manejo de la herramienta (movimiento, interacción con equipo y entorno).

6. Se le asigna al sujeto de prueba la misión que debe desarrollar en el entorno de realidad virtual. Se inicia la recolección de las señales biológicas y se graba el video de la misión que se ha asignado. La duración aproximada de la misión no puede superar los veinte minutos, tiempo máximo recomendado de inmersión en realidad virtual.

7. Una vez completado el ejercicio, se le solicita al sujeto de investigación diligenciar una encuesta para evaluar la experiencia virtual.

8. Las señales biológicas recolectadas son guardadas en formato de archivo plano (.csv), convertido y almacenado en formato matricial (.mat) para su posterior análisis.

\section{Resultados}

\section{Análisis de necesidades}

El simulador ha empezado a tener funciones importantes en la formación del personal en la industria militar. Entre las prácticas que realizan en este proceso se encuentra el área de tiro, la cual inicia desde principios básicos 
hasta tiradores de alta precisión. La realización de estos ejercicios requiere costos de munición, transporte, tiempo y en algunos casos se presentan accidentes, aspectos que son controlados en los ambientes de simulación (González et al., 2013).

Específicamente, la EsMIC implementó un simulador de tiro Beam Hit, un blanco computarizado que funciona por medio de un adaptador de energía de corriente alterna (CA) (BeamHit, 2008, 2010) y que debe contar con un tomacorriente en uso, así como con un espacio cerrado para no exponer el blanco computarizado a la lluvia o la humedad. Sin embargo, este sistema trae siluetas de tiro predefinidas, lo cual genera la percepción de ejercicios de tiro simulado carentes de realismo, pues no permite que el tirador esté en condiciones semejantes a las reales. Además, se evidencia la cadencia de disparo, que actualmente solo se permite tiro a tiro, de manera que el operador debe correr los mecanismos de su arma hacia atrás para realizar cada disparo.

Por su parte, la evaluación del liderazgo y la toma de decisiones se encuentra enfocada en el desarrollo de ejercicios prácticos en terreno mediante el entrenamiento como comandante de pequeńas unidades para el desarrollo de maniobras de combate irregular, las cuales se basan en el reglamento de operaciones en maniobras de guerra irregular y el manual del lancero (Comando General de las Fuerzas Militares, 2003).

Esta forma de entrenamiento presenta inconvenientes desde el punto de vista de la evaluación, ya que aproximadamente lo realizan de manera paralela 150 uniformados en proceso de formación, lo cual dificulta el seguimiento y la retroalimentación por parte del instructor desde el punto de vista militar. De igual manera, no existe una herramienta que permita evaluar el estado físico y mental del personal en formación ante una situación de acción sorpresiva.

\section{Fabricación de los sistemas de simulación}

Para el desarrollo del sistema de simulación orientado a la evaluación de tiro se parte de las falencias identificadas en los sistemas de tiro implementados actualmente (tabla 1$)$. 
Tabla 1. Falencias en el simulador de tiro implementado actualmente

\begin{tabular}{cl}
\hline Ítem & Falencia \\
\hline 1 & Conexión continua a red eléctrica. \\
\hline 2 & Sensibilidad a la electrostática, filtración en los cables de datos. \\
\hline 3 & $\begin{array}{l}\text { Arma y blanco independientes, de manera que el tirador le puede } \\
\text { disparar a cualquier blanco. }\end{array}$ \\
\hline 4 & Siluetas de acuerdo al país de fabricación. \\
\hline 5 & Control de disparos de acuerdo con el ejercicio. \\
\hline 6 & $\begin{array}{l}\text { Retroceso mediante aire comprimido o CO2 (gastos mensuales) o no } \\
\text { cuentan con esta característica. }\end{array}$ \\
\hline 7 & Mantenimiento y repuestos importados. \\
\hline
\end{tabular}

Fuente: Elaboración propia.

Con base en lo anterior se desarrolla un sistema que cuente con los elementos y principios de funcionamiento básicos de simuladores de tiro (Northropgrumman, 2020) (figura 2) y a su vez de solución a las necesidades y falencias previamente mencionadas.

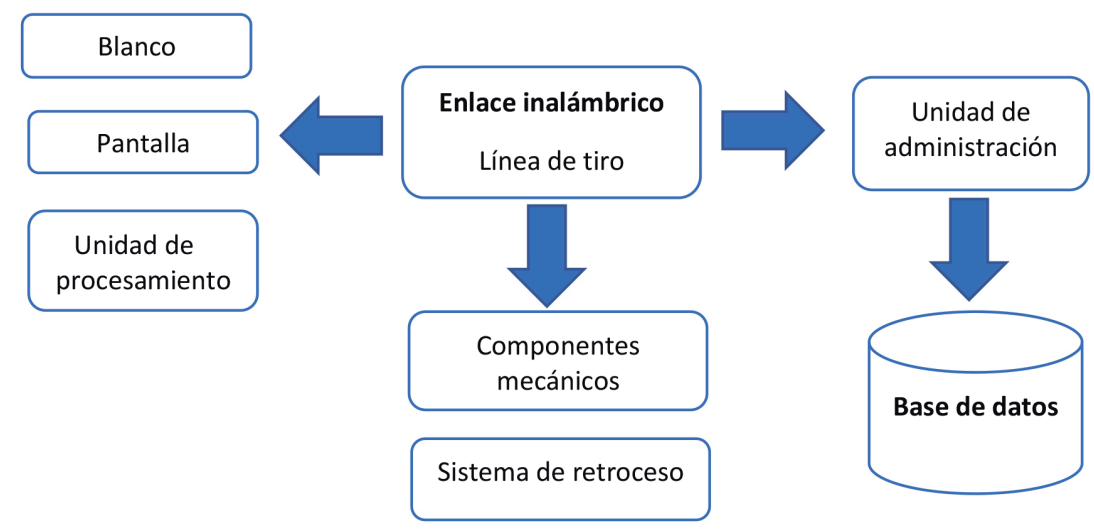

Figura 2. Principio de funcionamiento simulador de tiro.

Fuente: Elaboración propia. 
En los desarrollos realizados en la unidad de procesamiento, cuando se dispara, el sistema proyecta, mediante un láser, un punto luminoso en el blanco. El dispositivo de captura de imágenes consta de una cámara que monitorea constantemente el blanco. Posteriormente, la imagen capturada es procesada para detectar las coordenadas del centro del punto que representa el disparo, las cuales son enviadas al software, encargado de definir el puntaje y graficar la ubicación del disparo.

En cuanto a los componentes mecánicos, el sistema desarrollado cuenta con un mecanismo de retroceso (figura 3) con el objetivo de lograr la mayor similitud con una culata real del fusil Galil Ace 23 calibre $5,56 \mathrm{~mm}$, considerando el tamaño y el peso, pero que, a su vez, esta misma contenga los elementos necesarios para realizar el retroceso del fusil al momento de activar el disparador.

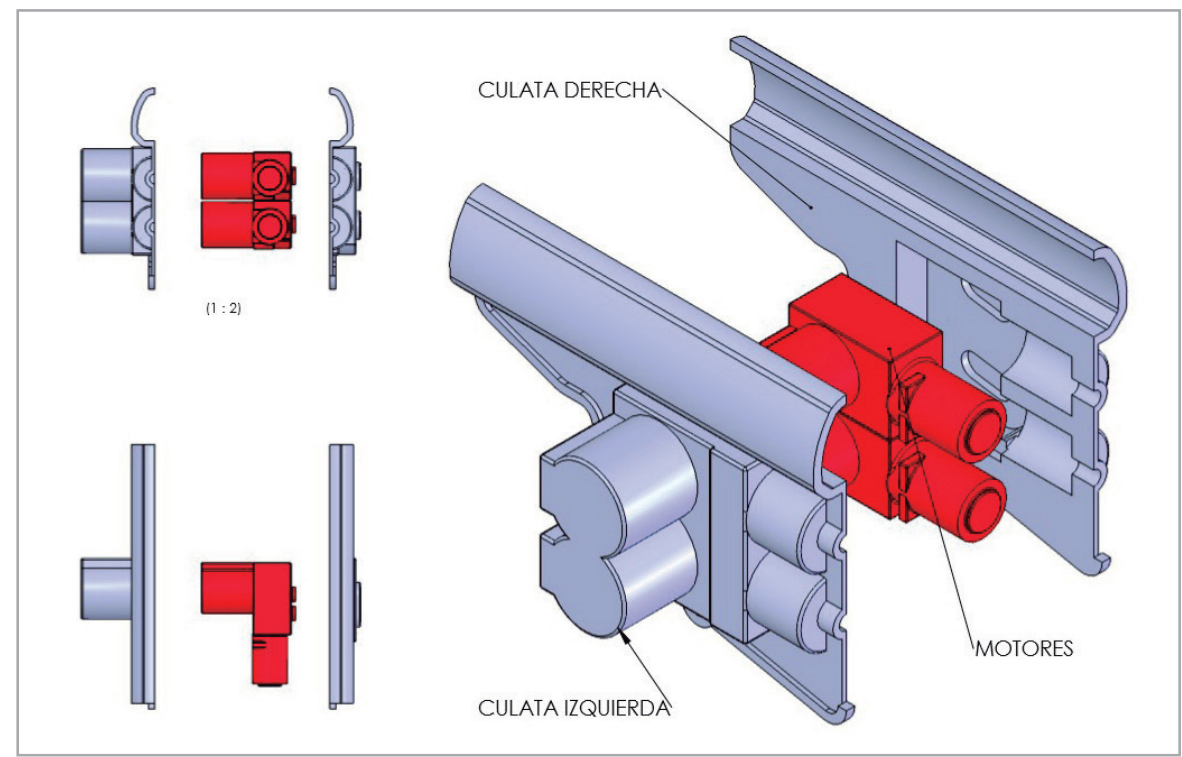

Figura 3. Mecanismo de retroceso.

Fuente: Elaboración propia.

En cuanto al software de administración (figura 4), es posible seleccionar los ejercicios establecidos por el Ejército Nacional para arma larga. Asimismo, permite la visualización de información en tiempo real y la generación de reportes personalizados. 

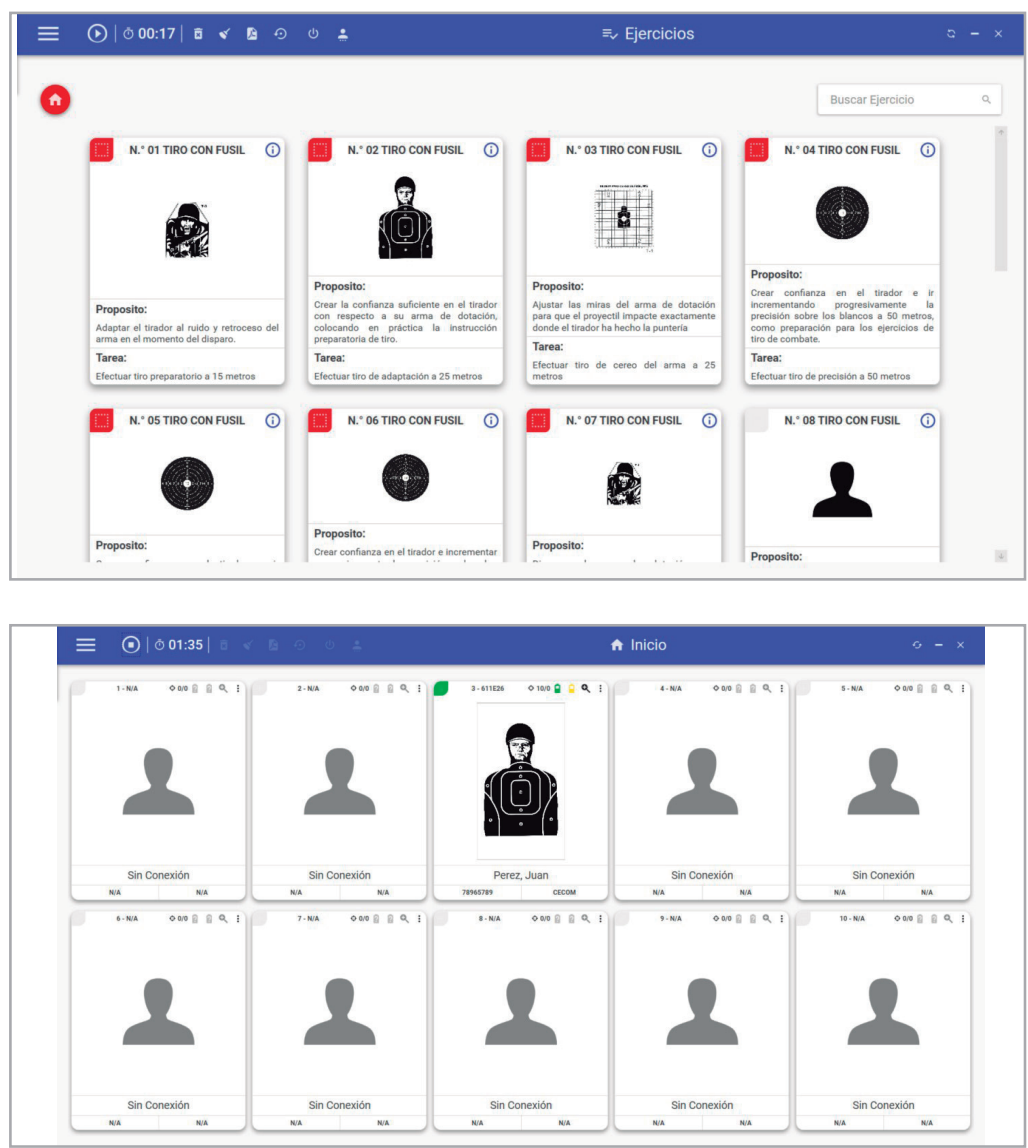

Figura 4. Software de administración. Fuente: Elaboración propia.

Este cuenta además con conexión wifi y sistemas de alimentación mediante el uso de baterías, lo que lo vuelve un sistema portable para su uso en las diferentes unidades militares del territorio nacional.

Por otra parte, para el desarrollo del sistema de evaluación de liderazgo y toma de decisiones se diseñó un casco para implementar la interfaz BCI (figura 5), teniendo en cuenta las siguientes indicaciones:

- Canaletas para el cableado de electrodos y las gafas HTC vive.

- Ubicación de electrodos.

- Ajuste de baterías y carcasa de tarjeta microcontroladora. 

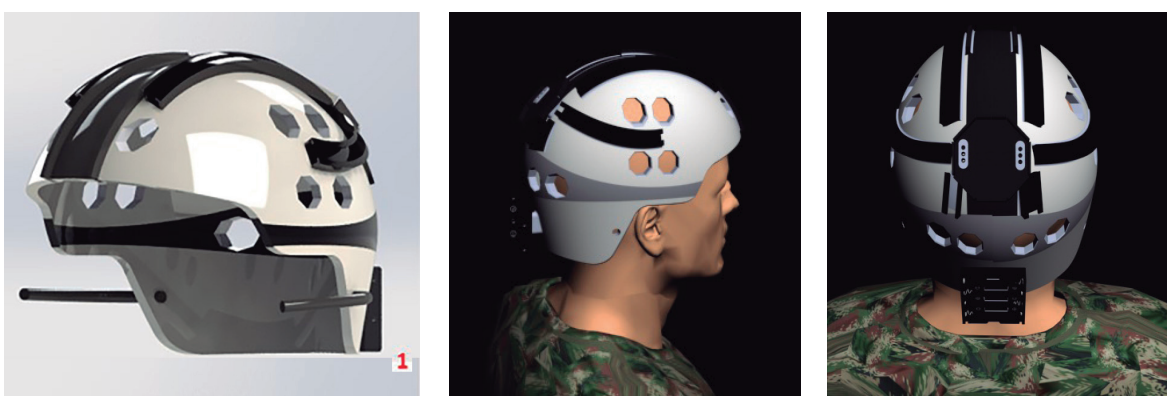

Figura 5. Diseńo del casco para monitorear la actividad cerebral. Fuente: Elaboración propia.

Una vez definido el diseño del casco, se fabricó en una impresora 3D (figura 6).

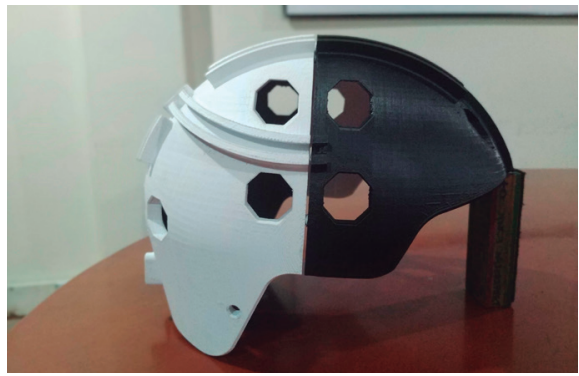

Figura 6. Impresión de casco en impresora 3D.

Fuente: Elaboración propia.

Posteriormente, se realizó la conexión de electrodos y la implementación de la interfaz cerebro-computadora BCI (figura 7).

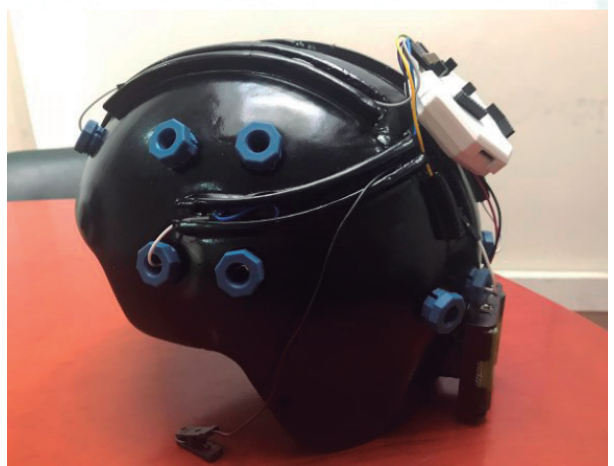

Figura 7. Interfaz cerebro-computadora BCI.

Fuente: Elaboración propia. 
Finalmente, se realizó la integración y prueba del casco desarrollado con el sistema de entrenamiento en entornos militares simulados en realidad virtual que existe en la EsMic.

\section{Pruebas de funcionalidad}

En cuanto al sistema que se desarrolló para evaluar la precisión de tiro siguiendo el protocolo previamente mencionado, se realizó una primera prueba con treinta sujetos de investigación en las instalaciones del Batallón Simón Bolívar N. 1 del departamento de Boyacá, ubicado en la ciudad de Tunja (figura 8).

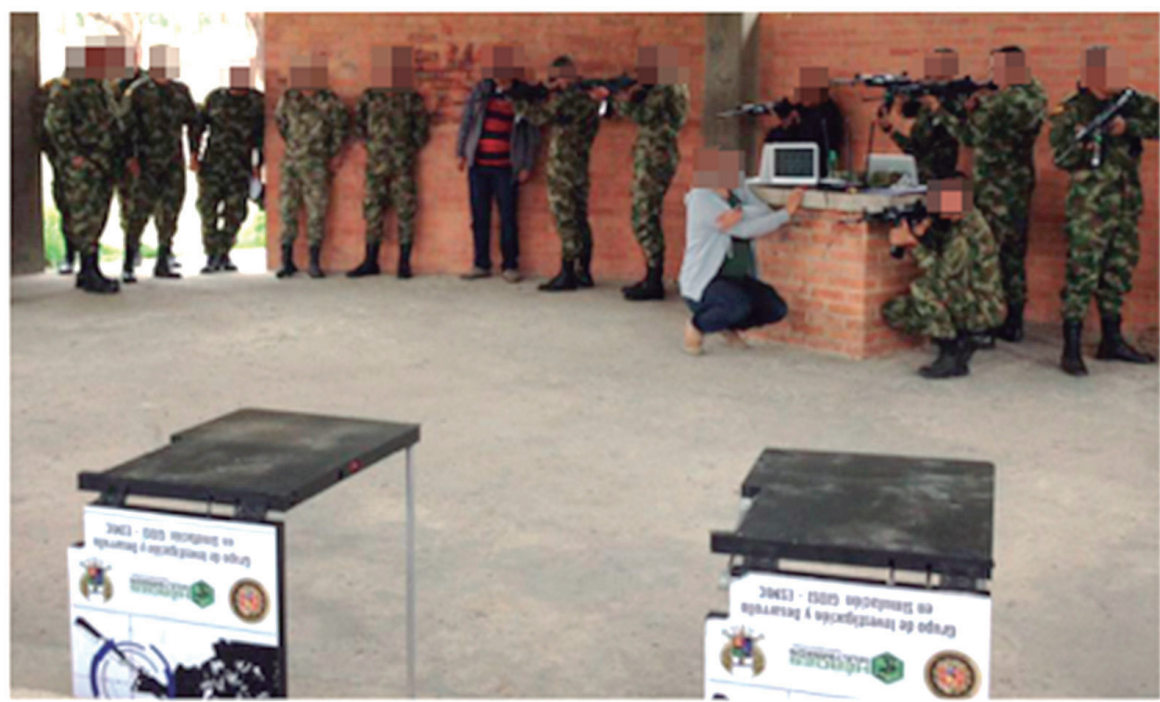

Figura 8. Pruebas de funcionalidad del sistema de tiro implementado. Fuente: Elaboración propia.

Al finalizar la prueba se realizó una encuesta de validación, para lo cual se utilizó un tamaño de muestra de treinta encuestados (figura 9): soldados profesionales, suboficiales u oficiales que han tenido experiencia en ejercicios de polígono real. 


\section{Pregunta 1}

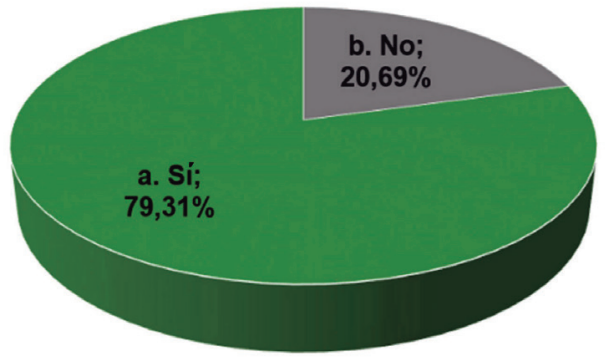

-b. No $=$ a. Sí

Figura 9.1. Pregunta 1: ¿Usted ha realizado ejercicios de tiro en sistemas de simulación?

\section{Pregunta 2}

c.Ligeramente más pequeña

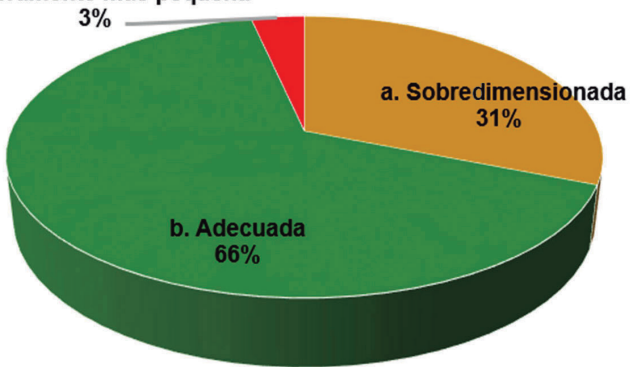

" a. Sobredimensionada $\quad$-b. Adecuada - c.Ligeramente más pequeña

Figura 9.2. Pregunta 2: Después de terminar la práctica de tiro, ¿̨usted considera que las dimensiones de los acoples son...?

\section{Pregunta 3}

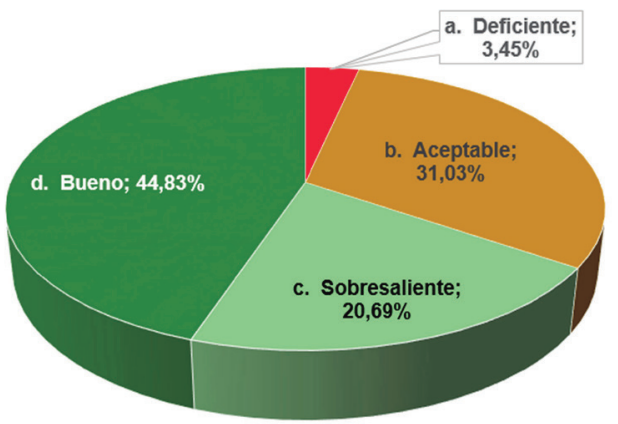

-a. Deficiente $\quad$ b. Aceptable $\quad$ c. Sobresaliente $\quad$ d. Bueno

Figura 9.3. Pregunta 3: Al terminar su práctica de tiro, ¿considera que el funcionamiento del sistema es...? 


\section{Pregunta 4}

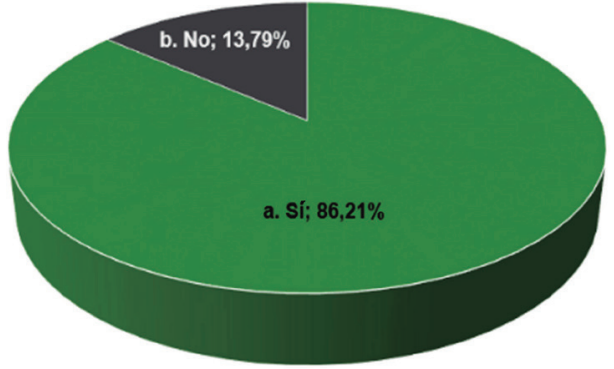

-a. Si $\quad$ b. No

Figura 9.4. Pregunta 4: ¿¿Usted considera adecuado el peso del arma con sus elementos adicionales?

\section{Pregunta 5}

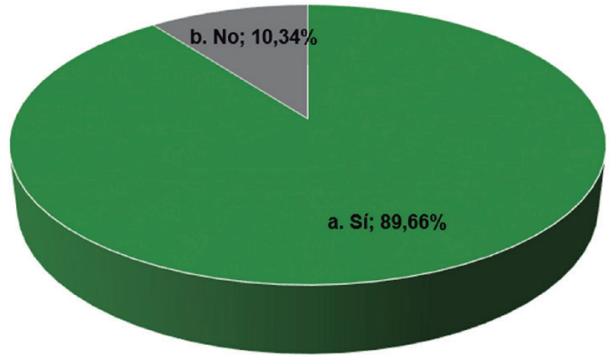

-a. Sí $\quad$ b. No

Figura 9.5. Pregunta 5: ¿Usted considera adecuado el funcionamiento del acople en el disparador?

\section{Pregunta 6}

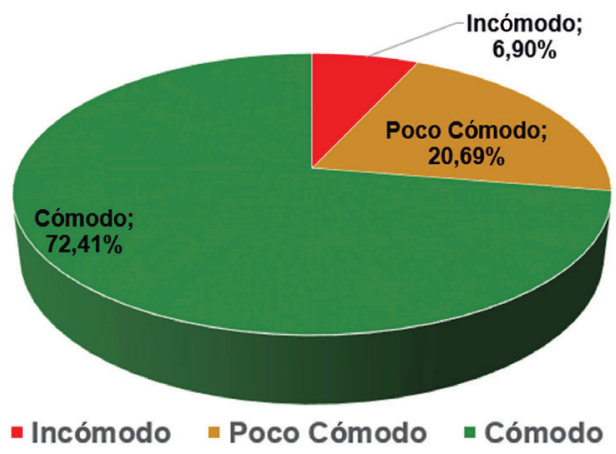

Figura 9.6. Pregunta 6: ¿Cómo percibe usted el agarre del fusil? 


\section{Pregunta 7}

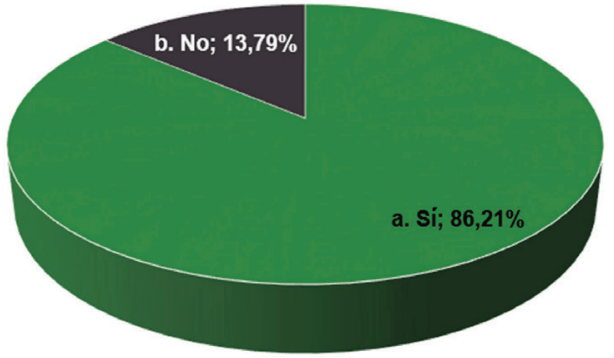

-a. Sí $\quad$ b. No

Figura 9.7. Pregunta 7: ¿Usted considera que el material empleado en los diversos elementos que se acoplan al fusil son los adecuados?

\section{Pregunta 8}

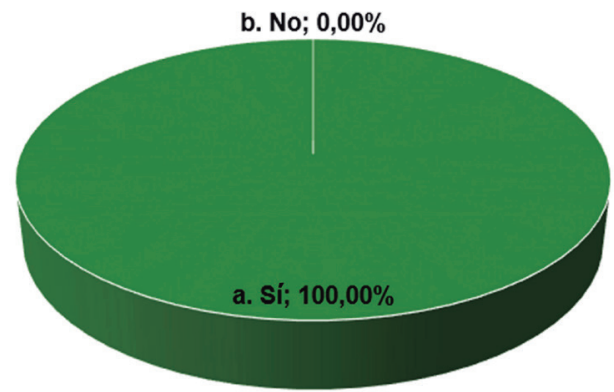

-a. Si $=$ b. No

Figura 9.8. Pregunta $8:$ ¿Le parece adecuado los colores empleados en el blanco portátil?

\section{Pregunta 9}

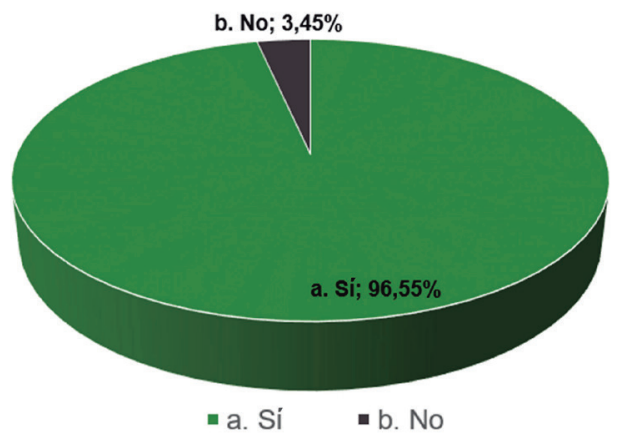

Figura 9.9. Pregunta 9: ¿Usted considera que el procedimiento realizado en la práctica de tiro es el idóneo? 


\section{Pregunta 10}

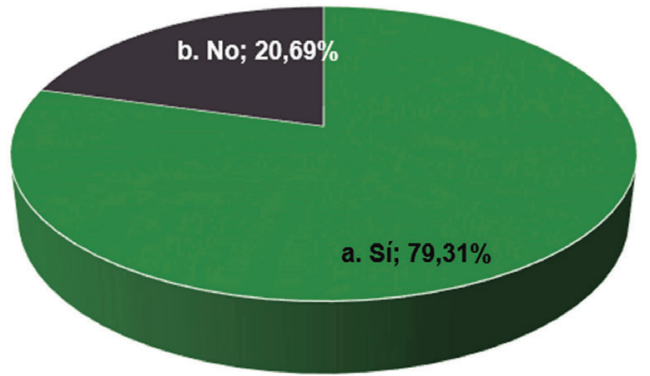

- a. Sí mb. No

Figura 9.10. Pregunta 10: ¿Usted considera que al terminar la práctica de tiro el puntaje que obtuvo demuestra sus falencias como tirador?

\section{Pregunta 11}

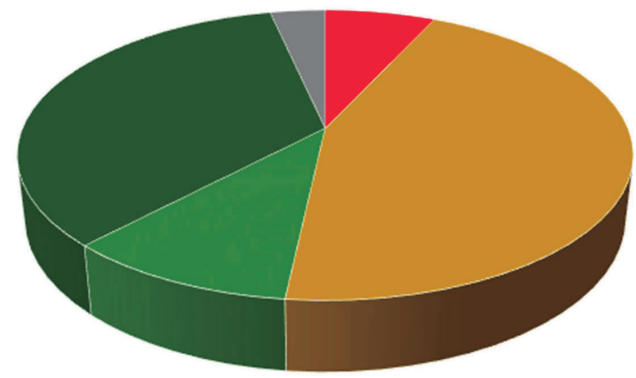
- a. Deficiente
$\because$ b. Aceptable
- c. Sobresaliente
- d. Buena
- e. Ninguna de las anteriores

Figura 9.11. Figura 11: ¿Qué tan parecido con un polígono real experimentó la práctica de tiro realizada en el polígono láser portátil fase 3.0?

Figura 9. Resultados de las encuestas de validación sistema de tiro.

Fuente: Elaboración propia.

Por otra parte, respecto al sistema que se desarrolló para evaluar el liderazgo y la toma de decisiones, se realizó una muestra de investigación de $\mathrm{N}=20$, se analizaron un estado base y cuatro acciones específicas basadas en la doctrina militar. Estas acciones estaban relacionadas con un ataque sorpresivo, del cual no tiene conocimiento el sujeto de investigación: "Período base: Una referencia en estado de reposo sin interacción con el sistema de realidad virtual". 
- Acción 1. El grupo subversivo embosca al equipo sujeto. La reacción, según la doctrina, es buscar cobertura y protección.

- Acción 2. El sujeto establece la base de fuego para poder maniobrar con unidades.

- Acción 3. El sujeto inicia su participación con unidades no comprometidas.

- Acción 4. La acción de reorganización e inspección grupal una vez finalizada la emboscada.

Mientras el sujeto de investigación se encuentra inmerso en la realidad virtual desarrollando la misión, se monitorea la actividad cerebral y la frecuencia cardiaca, y se graba todo el desarrollo de la misión (figura 10).
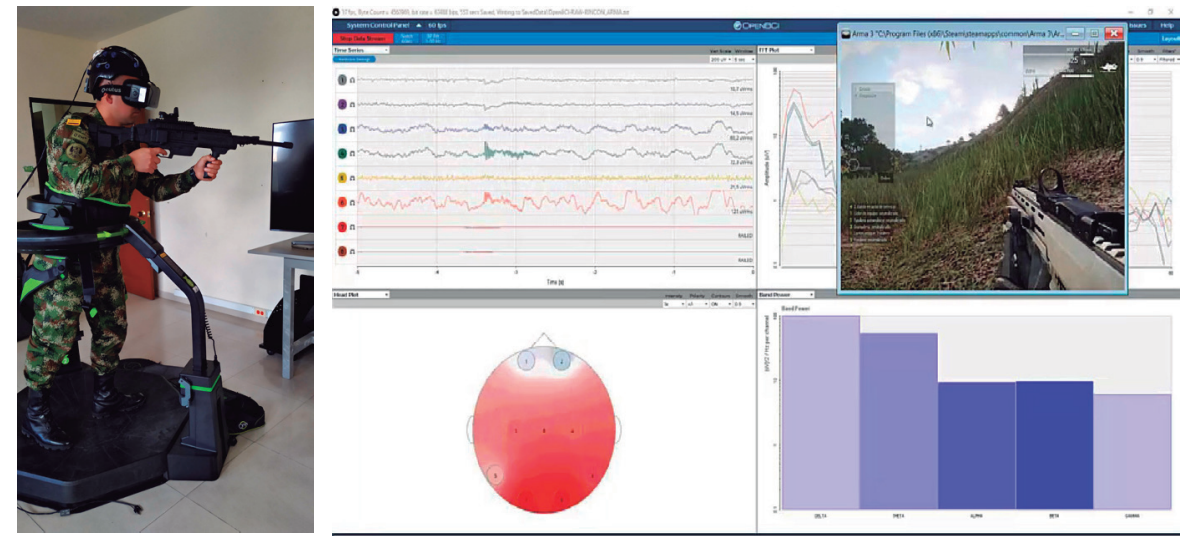

Figura 10. Prueba de funcionalidad.

Fuente: Elaboración propia.

Durante la realización del ejercicio se evidenció la adecuada adaptación y lectura del casco de actividad cerebral y el sensor de la frecuencia cardiaca, así como la adecuada interoperabilidad en la toma de datos en tiempo real. Igualmente, se evidenció que ninguno de los sujetos de investigación completó la misión. La información recolectada va a ser analizada desde el punto de vista de la ejecución militar y el desarrollo de redes neuronales complejas como trabajo de investigación futuro. 


\section{Discusión}

El análisis de los sistemas de simulación utilizados para la práctica de tiro permitió plantear las desventajas que estos presentan en el desarrollo del entrenamiento en la ESMIC y las soluciones implementadas en el sistema desarrollado en la investigación. Asimismo, se evidencian las mejoras con las que cuenta el sistema para la evaluación de precisión de tiro diseñado con respecto a los simuladores que se encuentran actualmente en la Fuerza.

De acuerdo con los resultados de las encuestas realizadas luego de las pruebas de funcionalidad en el simulador de tiro, es posible afirmar que el sistema permite una adecuada evaluación del desempeńo en práctica de tiro, con una calificación del $79 \%$ de aprobación. Asimismo, esta información muestra que el sistema se encuentra en un rango de aprobación entre aceptable, sobresaliente y bueno. Finalmente, se evidencian aspectos susceptibles de mejorar, como la comodidad y las dimensiones del sistema de agarre que se evidencia en las encuestas realizadas.

En cuanto al sistema desarrollado para el fortalecimiento y la evaluación del liderazgo y la toma de decisiones, los impactos están relacionados en mejorar el entrenamiento de militares encargados de efectuar maniobras de fuego bajo situaciones de estrés. Es posible afirmar que el sistema desarrollado funciona adecuadamente desde el punto de vista tecnológico para la recolección de información. Durante la realización del ejercicio se evidenció la adecuada adaptación y lectura del casco de actividad cerebral y el sensor de frecuencia cardiaca, así como la adecuada interoperabilidad en la toma de datos en tiempo real. De igual manera, se observó que ninguno de los sujetos de investigación completa la misión desde el punto de vista militar. La información recolectada va a ser analizada desde la perspectiva de la ejecución militar y la señal electroencefalográfica se analizará mediante el desarrollo de redes neuronales complejas como trabajo de investigación futuro.

\section{Conclusiones}

Dado el potencial beneficio que podrían generar las plataformas de simulación, la Escuela Militar de Cadetes "General José María Córdova” (ESMIC) continuará fortaleciendo el nivel de educación militar mediante el desarrollo y 
la implementación de esta tecnología, tanto para el entrenamiento individual en el área de tiro, como para la evaluación del liderazgo y el entrenamiento en la toma de decisiones en procedimientos tácticos en un ambiente de operaciones simulado.

Esta investigación ha permitido demostrar su pertinencia y la necesidad de implementar los diferentes simuladores en la ESMIC para el entrenamiento en las diferentes unidades militares del país. Además, a través de cada uno de los casos de estudio se han logrado resultados de alto impacto científico para la nueva generación de la educación en la Fuerza. Finalmente, también se ha logrado identificar trabajos futuros y aspectos por mejorar de los desarrollos tecnológicos desde la perspectiva del usuario final.

\section{Referencias}

Beamhit. (2008). Machine Gun Training System (MGTS). American ANSI-Z.

Beamhit. (2010). Manual del sistema 460. 58-59. Columbia, Estados Unidos.

Bera, T. K. (2015). Noninvasive electromagnetic methods for brain monitoring: A technical review. Intelligent Systems Reference Library, (74). https://doi.org/10.1007/978-3-31910978-7_3

Brash 3D. (2019). Estudio de factibilidad de un simulador de realidad virtual de entrenamiento y capacitación del vehículo militar Pegaso M1117 y torreta automatizada. https:/www. brash3d.com/

Ciro, A., \& Correa, M. (2014). Transformación estructural del Ejército colombiano: Construcción de escenarios futuros. Revista Cientifica General José María Córdova, 12(13), 19-88. https://doi.org/10.21830/19006586.155

Comando General de las Fuerzas Militares. (2003, julio 28). Disposición número 039 de 2003. Ministerio de Defensa Nacional.

Ejército Nacional de Colombia. (2015). Organigrama Ejército Nacional de Colombia. https:// www.ejercito.mil.co/?idcategoria $=27$

Ejército Nacional de Colombia, Departamento de Investigación, Ciencia, Desarrollo Tecnológico e Innovación. (2009, enero 12). Directiva 0038 de Investigación: Creación del Sistema de Ciencia y Tecnología del Ejército Nacional. Jefatura de Educación y Doctrina.

Ejército Nacional de Colombia, Jefatura de Educación y Doctrina. (2013, mayo 28). Directiva 0120. Sistema de Ciencia y Tecnología del Ejército. Dirección de Ciencia y Tecnología. Jefatura de Educación y Doctrina.

Elbit Systems. (2020). MAY System Deployable Solution. www.elbitsystems.com 
98 Volumen II. Avances y desafíos contemporáneos en la educación de las Fuerzas Armadas

Giroux, H. (1997). La pedagogía de frontera y la política del postmodernismo. Revista Intringulis, (6), 33-47.

González, W., Jaimes, U., \& Hernández, J. (2013). Prototipo de polígono virtual de adaptación. Escuela de Comunicaciones Militares.

Kenneth, E. K., \& Julie, E. K. (2017). Análisis y diseño de sistemas. Pearson Educación.

Margalef, L., \& Arenas, A. (2006). ¿Qué entendemos por innovación educativa? A propósito del desarrollo curricular. Perspectiva Educacional, 1(47), 13-31.

Northropgrumman. (2020). C4ISR Careers. www.northropgrumman.com

Roselli, N. (2011). Teoría del aprendizaje colaborativo y la teoría de la representación social: Convergencias y posibles articulaciones. Revista Colombiana de Ciencias Sociales, 2(2), 173-191.

Slavin, R. (2002). Aprendizaje cooperativo: Teoría, investigación y práctica. AIQUE. 